\section{Bright spark}

\section{New Astronomy: An International, Electronic Journal in Astronomy and Astrophysics \\ Editors Donald C. Backer et al. \\ Elsevier. 4/yr. \$460, NFl800 (institutional); $\$ 129$, NFl225 (personal) \\ http://www.elsevier.nl/locate/newast}

\section{Michael Rowan-Robinson}

When I started research in astronomy, I wrote my computer programs on punched cards, and there were plenty of people of the previous generation who declined to use computers at all. Today all is utterly changed and no-one can survive in the field without a fair degree of computer literacy. Electronic mail and the World-Wide Web have become powerful tools for communication and collaboration. The hard-copy preprint is heading for extinction. Will the hard-copy journal head in the same direction?

I guess the promoters of New Astronomy, who include an impressive international board of editors, must feel the answer to that question is "yes". For otherwise it might seem odd to launch a journal in a field well served by established journals. With Astrophysical Journal, Monthly Notices of the Royal Astronomical Society, Astronomy and Astrophysics and Astronomical Journal all of high quality, and several others regularly containing important papers, there does not at first sight seem much need for a new journal.

The unique feature of New Astronomy, however, is that it is essentially an electronic journal. There is a paper version too and submission on paper is permitted. But most of the strengths of the new journal relate to its primary electronic form. The text can be viewed on the Web in full text or 'snapshot' form; there are integrated links to the bibliography; hypertext links to astronomical databases for every astronomical object mentioned; colour figures and large databases included (in the electronic version) free of charge; and electronic search facilities.

Other benefits include no page charges, a short publication time (a month from acceptance to electronic publication) and, as a start-up bonus, an annual complimentary subscription to the paper edition for the author of each accepted article.

The test, though, is the quality and the volume of the papers published. To put it in a nutshell: do I need to browse this journal regularly? At the moment, New Astronomy is publishing about five articles a month, compared with about 70 a month in Astrophysical Journal, the leading astronomical journal. New Astronomy will have to get a lot bigger for astronomers to feel that their best papers need to published here. But the quality of the articles is certainly high and there are several papers I was pleased to have read.

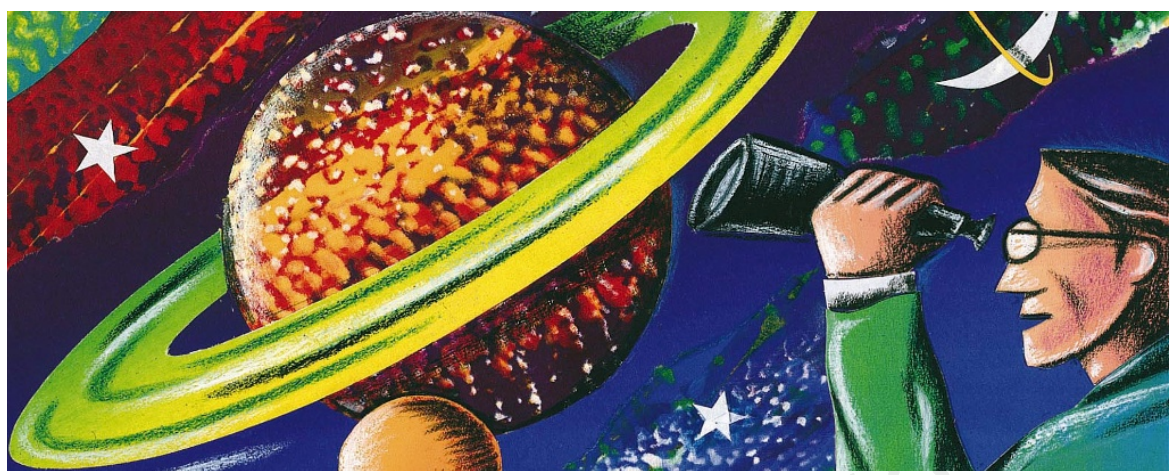

This brings me to what I certainly see as the principal drawback of the electronic journal. Personally, I hate reading extended texts on a screen. I find I cannot really take the information in without being able to flip backwards and forwards, seeing the shape of the whole. Computers are wonderful for storing and retrieving information. The Web would be wonderful if it wasn't so slow and unreliable. But I will always want to read articles and books in paper form. Long live Caxton!

New Astronomy is an interesting concept and will appeal to younger scientists. It is off to a good start and may be the journal of the future. It will, at least, trigger other journals into more electronically friendly habits. Michael Rowan-Robinson in the Astrophysics Group, Blackett Laboratory, Imperial College of Science, Technology and Medicine, Prince Consort Road, London SW7 2BZ, UK.

\section{Journal reincarnate}

\section{Astronomy and Geophysics: The Journal of the Royal Astronomical Society}

Editor Sue Bowler

Institute of Physics Publishing. 6/yr. Europe

£92, USA \$161, elsewhere $£ 102$

(institutional); Europe $£ 40$, North America

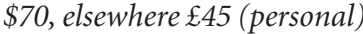

\section{Duncan Steel}

How can Astronomy and Geophysics $(A \& G)$ be a new journal when the initial issue announces itself to be the first of volume 38 ? The answer seems to be that some sleight of hand has taken place. With libraries everywhere cutting subscriptions, why not give your axe both a new head and a new handle, but insist that basically it is still the original? Certainly $A \& G$ has little in common with its predecessor (Quarterly Journal of the Royal Astronomical Society QJRAS), and it is really a magazine, with a striking format full of colour.

Such an attempt at legerdemain can backfire: the Royal Astronomical Society formerly received two-thirds of the costs of the QJRAS from outside subscriptions (as opposed to fellows' copies) and, although the charges remain on a par, what is to be supplied by $A \& G$ appears to be rather less, at least in terms of volume. My inclination on receiving the first few copies was to peruse them - and then file them in the bin rather than alongside the QJRAS on my bookshelf. The problem the society faces is whether librarians worldwide will act in the same way, resulting in cancellations. I hope not. It is easy to oppose change; but sometimes you dump an Edward VIII and get a George VI instead. incarnation: the Occasional Notes of the RAS (1938-59) were superseded in 1960 by volume one of the QJRAS, reaching volume 37 in 1996, and it is that journal (coalesced with the informal RAS Newsletter of recent years) that $A \& G$ now replaces. Other society journals have had similar reincarnations; witness the history of Geophysical Journal International, as related on its inside front cover. This also explains why geophysics appears in the title of $A \& G$, the discipline (both solid-Earth and atmospheric/space branches) having been long represented in the RAS membership, despite the society's motto, which exhorts members to study all that shines.

The contents of $A \& G$ seem to have settled down quickly: a few pages of news, a few of views, then about 20 pages of features (brief, accessible articles and reviews on specialized topics, refereed at some level and carrying referencelists), a few pages of book reviews, a bit about happenings within the RAS, a couple of obituaries, and a diary of events. Forty pages in all, including the covers. The muchlonger QJRAS owed its bulk to its extensive scientific discussion papers; perhaps these will now find a home in the Monthly Notices of the RAS, which is going stronger than ever after 170 years, necessitating - despite its title - twice-monthly publication.

$A \& G$ is colour-printed on glossy paper throughout, so impecunious authors can present the vivid graphics (so common in astronomical and geophysical work) that prohibitive page charges for most journals tend to exclude. Another attraction is speed of publication: no waiting a year or so here. But $A \& G$ must aim to be a journal of record, so one would hope that the several misstatements noticeable in the first issues - some trivial, but others egregious - will become less frequent as the publication matures. $\square$ Duncan Steel is at Spaceguard Australia P/L, PO Box 3303, Rundle Mall, Adelaide, SA 5000, Australia.
In fact, this is the publication's third 